\title{
An Efficient Algorithm for Mining String Databases under Constraints *
}

\author{
Sau Dan Lee Luc De Raedt \\ Institute for Computer Science, University of Freiburg, Germany \\ \{danlee, deraedt\}@informatik.uni-freiburg.de
}

\begin{abstract}
We study the problem of mining substring patterns from string databases. Patterns are selected using a conjunction of monotonic and anti-monotonic predicates. Based on the earlier introduced version space tree data structure, a novel algorithm for discovering substring patterns is introduced. It has the nice property of requiring only one database scan, which makes it highly scalable and applicable in distributed environments, where the data are not necessarily stored in local memory or disk. The algorithm is experimentally compared to a previously introduced algorithm in the same setting.
\end{abstract}

\section{Introduction}

In recent years, the number of string databases (particularly, in bioinformatics) has grown enormously [1. One of the motivations for constructing and maintaining these databases is the desire to discover new knowledge from these databases using data mining techniques. While more traditional data mining techniques, such as frequent itemset mining [2] and frequent sequence mining [3], can be adapted to mine string databases, they do not take advantage of some properties specific to strings to accelerate the mining process. By specifically targeting string databases, it should be possible to devise more effective algorithms for discovering string patterns.

The most important contribution of this paper is the introduction of a novel algorithm, called FAVST, for mining string patterns from string databases. This algorithm combines ideas from data mining with string processing principles. More specifically, we employ ideas from suffix trees 415 to represent and compute the set of patterns of interest. The data structure used is that of Version Space Trees (VST, introduced by [6) to organize the set of substring patterns being discovered. We have observed that a suffix trie can be treated as a deterministic automata so that we can visit all the substring patterns contained in a data string efficiently. We exploit this property of the suffix trie in VST and devised the FAVST algorithm (see Sect. 5.2.). This algorithm performs frequency counting in only one database scan. It is thus is especially efficient when database access is slow (e.g. over the internet). We also compare FAVST with a more traditional

\footnotetext{
* This work was supported by the EU IST FET project cInQ, contract number IST2000-26469.
} 
level-wise data mining algorithm, called VST, that we developed earlier 6. As it employs the same data structure VST and the same setting as FAVST, this provides an appropriate setting for comparison. Although FAVST consumes more memory than VST our experiments (Sect.6) show that the memory requirements are relatively cheap by today's hardware standards. Furthermore, as we will show, it can be controlled by imposing an upper bound on the length of patterns to be discovered, making FAVST very attractive in practice.

The two algorithms FAVST and VST discover all patterns that satisfy a conjunctive constraint or inductive query of the form $A_{1} \wedge \ldots \wedge A_{m} \wedge M_{1} \wedge \cdots \wedge M_{n}$ where the $A_{i}$ 's and $M_{j}$ 's are anti-monotonic and monotonic predicates, respectively.

\subsection{Related Works}

The present work is to a large extent motivated by the earlier MolFea system 788 , in which conjunctive queries (over anti-monotonic and monotonic predicates) for molecular features were solved using a version space approach. MolFea features are essentially strings that represent sequences of atoms and bonds.

On the theoretical side, we have previously introduced a general theory 619 for mining of general patterns (not restricted to strings) satisfying a complicated predicate, which is composed of anti-monotonic and monotonic ones using Boolean algebra (negation, conjunction and disjunction). In this theoretical framework, one key component is an algorithm for efficiently discovering patterns satisfying the conjunction of a set of anti-monotonic and monotonic predicates. It is this component that we are addressing in this paper.

Furthermore, we have also generalized the concept of version spaces for performing usual set operations [10. This results in an algebraic framework for handling general predicates composed of anti-monotonic and monotonic predicates.

There has been a lot of work in frequent itemset mining under constraints. In 11, a method for combining monotone and anti-monotone constraints is presented. In 12, the database is filtered and compressed into a compact prefix tree structure called the FP-tree and then frequent itemsets are computed from directly from the data structure. This is not to be confused with our approach, in which we base on a general theoretical framework that works not only itemsets, but also many other pattern spaces. We do not only mine frequent patterns, but any patterns in the pattern space that satisfy a conjunction of monotonic and antimonotonic constraints. Moreover, our implemenation uses a suffix tree rather than a prefix tree. The tree stores the string patterns being mined, rather than (filtered) data items from the database. In [13, the use of the FP-tree with several monotonic and anti-monotonic constraints is discussed. However, that approach is specific for itemset patterns.

This work is also related to that of 14, which addresses the same setting as VST and FAVST, i.e. mining strings under conjunctive constraints using the VST data structure. However, whereas VST and FAVST aim at minimizing the 
number of scans of the string database, the approach of 14 aims at minimizing the number of tests whether a string pattern covers an example. To this aim, 14] employs cost functions and the resulting algorithm is entirely different. Also, the algorithm by [14 is targeted at situations where testing whether a pattern covers an example is computationally expensive and the underlying data sets relatively small. These situations arise in e.g. graph mining, cf. [7.

The rest of this paper is organized as follows. Important definitions are introduced in Sect. 2. We take a closer look into the search space of the problem in Sect. 3 and describe a data structure to handle it in Sect. 4. Two algorithms are devised to construct this data structure. They're presented in Sect. 5. Our approach are verified by experiments presented in Sect. 6. Finally, we come up with conclusions in Sect. 7 .

\section{Definitions}

\section{$2.1 \quad$ Strings}

Definition 1. A string $s$ over an alphabet $\Sigma$ is a sequence of symbols from $\Sigma$. The length of a string $s$, denoted $|s|$, is the number of symbols in that sequence. The unique string of length zero is called the empty string, denoted $\epsilon$. The set of all strings over an alphabet $\Sigma$ is denoted $\Sigma^{*}$.

Definition 2. A substring $s^{\prime}$ of $s$ is a sequence of consecutive symbols taken from $s$. We denote this relation as $s^{\prime} \sqsubseteq s$. We also say that $s$ is a superstring of $s^{\prime}$, or $s \sqsupseteq s^{\prime}$.

Example 3. With an alphabet of $\Sigma=\{\mathrm{a}, \mathrm{b}, \mathrm{c}, \mathrm{d}\}$, the following sequences are valid strings: $\epsilon$, "ab", "abac", "dbacd". But "ae" is not a valid string over this alphabet, as e $\notin \Sigma$.

Note that $\sqsubseteq$ is a partial order relation. This fact is exploited in the design of our algorithms in Sect. 5 .

\subsection{Database and Substring Patterns}

Since our goal is to mine substring patterns from a database, we have to define these two terms first. Further, not all substring patterns are interesting. We express the interestingness of the patterns using a predicate. Patterns not satisfying the predicate are considered to be uninteresting, and hence should not to be generated.

Definition 4. A database $D$ over an alphabet $\Sigma$ is a bag (i.e. multi-set) of strings over $\Sigma$.

Definition 5. A pattern $s$ over an alphabet $\Sigma$ is a string over $\Sigma$.

Definition 6. A predicate $\mathcal{P}$ for substring patterns over $\Sigma$ is a boolean function on a substring pattern $s \in \Sigma^{*}$ and (sometimes) a database $D$. 
We include $D$ into the definition of predicates because our focus is mining patterns in databases, although it turns out that database-independent predicates are also useful in expressing the interestingness more precisely. In this latter case, we can simply treat $D$ as a dummy parameter of our predicate. When $D$ is a dummy parameter, we omit it for brevity.

Definition 7. Define two predicates:

$$
\begin{aligned}
\text { substring_of }(s ; t) & \equiv s \sqsubseteq t \\
\text { superstring_of }(s ; t) & \equiv s \sqsupseteq t
\end{aligned}
$$

where $t \in \Sigma^{*}$ is a constant string.

Example 8. Using $\Sigma=\{$ a, b, c, d $\}$, substring_of(ab; abc) and superstring_of(bcd; $\mathrm{bc})$ evaluate to true whereas substring_of $(\mathrm{cd} ; \mathrm{abc})$ and superstring_of $(\mathrm{b} ; \mathrm{bc})$ evaluate to false.

Note that a predicate may have other parameters, such as a constant string as illustrated above, or a frequency threshold as shown below. We require these extra parameters to be independent of the database $D$, so that they can be fitted into our framework by the syntactic transformation $\mathcal{P}\left(s, D ; x_{1}, x_{2}, \ldots, x_{k}\right) \mapsto$ $\mathcal{P}_{x_{1}, x_{2}, \ldots, x_{k}}(s, D)$.

Analogous to frequent itemset mining, we may express our interestingness in frequent substring patterns by imposing a minimum occurrence frequency.

Definition 9. Given a database $D$ over an alphabet $\Sigma$ and a pattern string $s \in \Sigma^{*}$, we define the frequency freq $(s ; D)$ to be the number of strings in $D$ that is a superstring of s. i.e.

$$
\operatorname{freq}(s, D)=|\{d \in D \mid s \sqsubseteq d\}|
$$

We define two predicates related to frequency.

Definition 10. Given a database $D$ and an integer $\theta$, define

$$
\begin{aligned}
& \operatorname{minimum}_{-} \text {frequency }\left(s, D ; \theta_{\text {min }}\right) \Longleftrightarrow \operatorname{freq}(s, D) \geq \theta_{\min } \\
& \text { maximum_frequency }\left(s, D ; \theta_{\max }\right) \Longleftrightarrow \operatorname{freq}(s, D) \leq \theta_{\max }
\end{aligned}
$$

When context is clear, we omit $s$ and $D$ and simply write minimum_frequency $(\theta)$ and maximum_frequency $(\theta)$.

Example 11. Let $\Sigma_{1}=\{\mathrm{a}, \mathrm{b}, \mathrm{c}, \mathrm{d}\}$ and $D=\{\mathrm{abc}, \mathrm{abd}, \mathrm{cd}, \mathrm{d}, \mathrm{cd}\}$. With this database, we have freq $(\mathrm{abc})=1$, freq $(\mathrm{cd})=2$, freq $(\mathrm{c})=3$, freq $(\mathrm{abcd})=$ 0 . And trivially, freq $(\epsilon)=|D|=5$. Thus, the following predicates evaluate to true: minimum_frequency $(c, D ; 2)$, minimum_frequency $(c d, D ; 2)$, maximum frequency $(\mathrm{abc}, D ; 2)$, maximum_frequency $(\mathrm{cd}, D ; 2)$.

In some applications (e.g. MolFea [7), it is useful to partition the database $D$ into different subsets $D_{1}, \ldots, D_{n}$ and define frequency predicates that counts only a subset of $D$. e.g. $\mathcal{A}_{1}=$ minimum_frequency $\left(s, D_{1} ; \theta_{1}\right)$ and $\mathcal{M}_{2}=$ maximum frequency $\left(s, D_{2} ; \theta_{2}\right)$. Then, we can construct a compound predicate $\mathcal{P}=\mathcal{A}_{1} \wedge \mathcal{M}_{2}$ to mine the patterns that are frequent in the subset $D_{1}$ but not in $D_{2}$. Our experiments in Sect. 6 make use of such a setting extensively. 


\subsection{The Substring Mining Problem}

Definition 12. Given an alphabet $\Sigma$, a database $D$, and a predicate $\mathcal{P}$, the problem of Mining Substring Patterns is to find the set of substring patterns over $\Sigma$ satisfying $\mathcal{P}$ :

$$
\operatorname{Sol}\left(\mathcal{P}, D, \Sigma^{*}\right)=\left\{s \in \Sigma^{*} \mid \mathcal{P}(s ; D)\right\}
$$

Example 13. Continuing from Example 11, let $P_{1}(s, D) \equiv$ minimum_frequency $(s$, $\left.D_{1} ; 2\right) \wedge$ superstring_of $(s ; \mathrm{d})$. Then, we have $\operatorname{Sol}\left(\mathcal{P}_{1}, D_{1}, \Sigma_{1}^{*}\right)=\{\mathrm{d}, \mathrm{cd}\}$.

\section{The Search Space}

To solve the problem of Mining Substring Patterns, one naïve approach is of course a brute-force search: check all the substrings over $\Sigma^{*}$ against $\mathcal{P}$ and print out the satisfying ones. However, since $\Sigma^{*}$ is countably infinite, one can never exhaust the whole pattern space, although one can enumerate them in a certain order.

A much better idea, as in itemset mining, is to exploit the structure of the search space. It has already been mentioned in Sect. 2.1 that $\sqsubseteq$ is a partial order relation. We will restrict the predicates to one of the following two types, or a conjunction of any number of them.

Definition 14. An anti-monotonic predicate $\mathcal{A}$ is a predicate that satisfies:

$$
\forall s_{1}, s_{2} \in \Sigma^{*} \text { such that } s_{1} \sqsubseteq s_{2}, \quad \mathcal{A}\left(s_{2}\right) \Rightarrow \mathcal{A}\left(s_{1}\right)
$$

Definition 15. A monotonic predicate $\mathcal{M}$ is a predicate that satisfies:

$$
\forall s_{1}, s_{2} \in \Sigma^{*} \text { such that } s_{1} \sqsubseteq s_{2}, \quad \mathcal{M}\left(s_{1}\right) \Rightarrow \mathcal{M}\left(s_{2}\right)
$$

Example 16. substring_of and minimum_frequency are anti-monotonic predicates whereas superstring_of and maximum_frequency are monotonic predicates.

With a compound query $\mathcal{P}=\left(\mathcal{A}_{1} \wedge \cdots \wedge \mathcal{A}_{m}\right) \wedge\left(\mathcal{M}_{1} \wedge \cdots \wedge \mathcal{M}_{k}\right)$, we can rewrite it as $\mathcal{P}=\mathcal{A} \wedge \mathcal{M}$, where $\mathcal{A}=\mathcal{A}_{1} \wedge \cdots \wedge \mathcal{A}_{m}$ and $\mathcal{M}=\mathcal{M}_{1} \wedge \cdots \wedge \mathcal{M}_{k}$. Note that $\mathcal{A}$ is anti-monotonic and $\mathcal{M}$ is monotonic. Therefore, we only need to consider predicates of the form $\mathcal{A} \wedge \mathcal{M}$.

While confining ourselves to predicates of this form may appear restrictive, we should note that in most formulations of data mining problems in the past years, an even more restrictive form of the predicate is used. For example, in most frequent-itemset, frequent-sequence mining problems, only a minimumfrequency predicate (anti-monotonic) is used. The consideration of using monotonic predicates has appeared only recently, and is still a rarity. [15]16] The general conjunction of an arbitrary number of monotonic and anti-monotonic predicate is seldom seen, either. Thus, our restricted form $\mathcal{P}=\mathcal{A} \wedge \mathcal{M}$ is already quite expressive. 
In previous works 69, we suggested how to support queries that are arbitrary boolean functions of anti-monotonic and monotonic predicates. As shown in those papers, mining patterns under of these arbitrary boolean predicates can be reduced to the mining of predicates of the form $\mathcal{P}=\mathcal{A} \wedge \mathcal{M}$ as well as some set manipulation operations. The latter can be done efficiently (see Sect. 4.1). The former is non-trivial, and is the most time-consuming step. So, in this paper, we concentrate on the algorithms and performance issues, and restrict ourselves mainly to the form $\mathcal{P}=\mathcal{A} \wedge \mathcal{M}$,

\subsection{Version Space}

Restricting the predicate to the form $\mathcal{P}=\mathcal{A} \wedge \mathcal{M}$, the set of solutions to the Subsection Mining Problem $\operatorname{Sol}\left(\mathcal{P}, D, \Sigma^{*}\right)$ turns out to be a version space 17 under the $\sqsubseteq$ relation. This means that there exists two sets $S, G \subseteq \Sigma^{*}$ with the following properties.

$-S=\left\{p \in \operatorname{Sol}\left(\mathcal{P}, D, \Sigma^{*}\right) \mid \nexists q \in \operatorname{Sol}\left(\mathcal{P}, D, \Sigma^{*}\right)\right.$ such that $\left.p \sqsubseteq q\right\}$

- $G=\left\{p \in \operatorname{Sol}\left(\mathcal{P}, D, \Sigma^{*}\right) \mid \nexists q \in \operatorname{Sol}\left(\mathcal{P}, D, \Sigma^{*}\right)\right.$ such that $\left.q \sqsubseteq p\right\}$

- $\forall p \in \operatorname{Sol}\left(\mathcal{P}, D, \Sigma^{*}\right), \exists s \in S \wedge g \in G$ such that $g \sqsubseteq p \sqsubseteq s$

$-\forall p, q, r \in \Sigma^{*}$ such that $p \sqsubseteq q \sqsubseteq r$, we have: $p, r \in \operatorname{Sol}\left(\mathcal{P}, D, \Sigma^{*}\right) \Rightarrow q \in$ $\operatorname{Sol}\left(\mathcal{P}, D, \Sigma^{*}\right)$

Example 17. For $\operatorname{Sol}\left(\mathcal{P}_{1}, D_{1}, \Sigma_{1}^{*}\right)$ from Example 13, we have $S=\{\operatorname{cd}\}$ and $G=$ $\{d\}$.

The set $S$ is called the maximally specific set and $G$ is called the maximally general set. For more details on how this mining problem relates to version spaces, please refer to our previous works 6]9. In this paper, we focus on the algorithms and optimizations.

\section{Version Space Trees}

To facilitate mining of string patterns, we have devised a data structure, which we called the version space tree (VST). We have already described the VST in other publications 69. So, we will give a brief overview of it here.

The VST data structure is inspired by the suffix tree, which is well studied 415. Instead of using a suffix tree, the VST is based on a less compact form, called suffix trie.

A trie is a tree with each edge labelled with a symbol from the alphabet $\Sigma$ concerned. Moreover, the labels on every edge emerging from a node must be unique. Each node $n$ in a trie thus uniquely represents the string $s(n)$ containing the characters on the path from the root $r$ to the node $n$. The root node itself represents the empty string $\epsilon$.

A suffix trie is a trie with the following properties:

- For each node $n$ in the trie, and for each suffix $t$ of $s(n)$, there is also a node $n^{\prime}$ in the trie representing $t$, i.e. $t=s\left(n^{\prime}\right)$. 
- Each node $n$ has as a suffix link suffix $(n)=n^{\prime}$ where $s\left(n^{\prime}\right)$ is the suffix obtained from $s(n)$ obtained by dropping the first symbol. Note that $\left|s\left(n^{\prime}\right)\right|=$ $|s(n)|-1$. The root node is special because it represents $\epsilon$, which has no suffixes. We define $\operatorname{suffix}($ root $)=\perp$, where $\perp$ is a virtual node, acting as a null pointer.

Example 18. The VST for $\operatorname{Sol}\left(\mathcal{P}_{1}, D_{1}, \Sigma_{1}^{*}\right)$ from Example13 is depicted in Fig.11. The numbers in each node $n$ shows freq $\left(s(n), D_{1}\right)$. The label of each node is shown to the left of the node. The dashed arrows show the suffix links. The suffix links of the first level of nodes have been omitted for clarity. They all point to the root node. Note that this diagram is for illustrative purpose. In practice, we would prune away all branches containing only $\ominus$ nodes to save memory.

What makes VST unique is that we make two major deviations from the main stream approach in the suffix tree culture. The first one is that instead of building a suffix trie on all the suffixes of a single string, we are indexing all the suffixes of a set of strings in a database $D$. This means multiple strings are stored in the tree. As intermediate computation results, we even keep a count of occurrences of each such substring. In addition to a count, we also store a label on each node of the VST. The label $\oplus$ indicates that the represented string pattern is in our solution $\operatorname{Sol}\left(\mathcal{P}, D, \Sigma^{*}\right)$. Otherwise, it is $\ominus$. Our algorithms in Sect. 5 exploit this label to store intermediate mining results. By contrast, theoretical works in the literature normally handle multiple strings by reducing the problem to a single string formed from concatenating the original strings, using a new delimiter symbol. While this is elegant in theory, it is impractical.

A second difference is that most studies on suffix trees usually consider a more compact form of suffix trie in which a chain of nodes with only one out-going edges are coalesced into one edge label with the string containing the symbols involved. We are not using this representation, as our algorithms need to keep flags and counts with each substring represented in the tree.

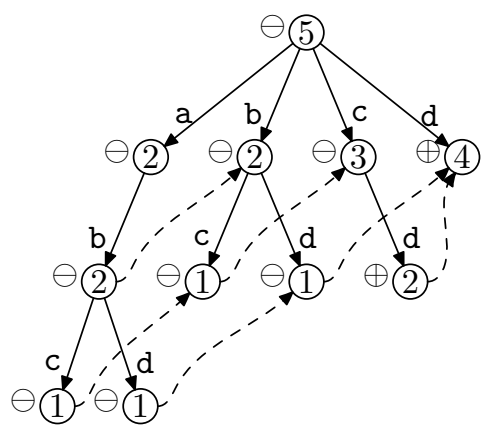

Fig. 1. A Version Space Tree 
One interesting property of a VST tree is that one can compute the sets $S$ and $G$ (see Sect. 3.1) easily by a tree traversal. The $S$ set consists of the strings $s(n)$ represented by the $\oplus$ nodes $n$ who have no $\oplus$ descendants and no other $\oplus$ nodes $n^{\prime}$ with suffix $\left(n^{\prime}\right)=n$. The $G$ set consists of the strings represented by the $\oplus$ nodes $n$ whose parent is $\ominus$ and $\operatorname{suffix}(n)$ is labelled $\ominus$.

Example 19. It can be easily checked from Fig. 1 with the above method that for $\operatorname{Sol}\left(\mathcal{P}_{1}, D_{1}, \Sigma_{1}^{*}\right)$ from Example 13. $S=\{\mathrm{cd}\}$ and $G=\{\mathrm{d}\}$. This is consistent with Example 17

\subsection{Beyond Version Spaces}

The algorithms to be presented in Sect. 5] are designed to build VSTs that represent sets of strings that form a version space under $\sqsubseteq$. This restriction to version spaces is only needed for the algorithms to build the VSTs efficiently. Once the trees are built, we can perform other operations on them, treating the VSTs as representations of sets only.

Indeed, once the VSTs are built, we can use tree merging algorithms to compute the unions, intersections and set differences of them. No access to the database $D$ is needed for these operations. Therefore, they can be performed completely in memory. We will see in section 6.4 that these operations generally takes little time when compared to the VST building, because the latter involves database scans.

With the possibility of performing general set operations on the resulting VSTs, we can actually handle more complicated predicates than the form $\mathcal{P}=$ $\mathcal{A} \wedge \mathcal{M}$. The problem is how to break down a general predicate $\mathcal{P}$ into subpredicates of the form $\mathcal{P}_{i}=\mathcal{A}_{i} \wedge \mathcal{M}_{i}$, so that $\operatorname{Sol}\left(\mathcal{P}, D, \Sigma^{*}\right)$ can be computed from the intermediate results $\operatorname{Sol}\left(\mathcal{P}_{i}, D, \Sigma^{*}\right)$ using set operations (e.g. union, intersection, set difference). We have made an elaborated study on this problem in previous publications 699. So, we are not repeating it here. In this paper, we give the details of the VST algorithm, as well as a new, faster algorithm FAVST.

\section{The Algorithms}

In this section, we present two algorithms to build a version space tree, given as input an alphabet $\Sigma$, a database $D$, and a predicate $\mathcal{P}$ of the form $\mathcal{A} \wedge \mathcal{M}$. Algorithm VST is a level-wise algorithm based on the well-known Apriori 18 algorithm. It was first introduced in 6. The other algorithm, FAVST is new and is based on techniques in the suffix-tree literature 4, which is much faster when the database size is large and the database access time is not negligible.

We require that $\mathcal{A}$ be a non-trivial anti-monotonic predicate 1 , as the version space and hence the corresponding tree with a trivial $\mathcal{A}$ will be infinite: There would be a finite integer $k$ such that all strings on $\Sigma$ with length greater than $k$ will satisfy $\mathcal{M}$. There is no such restriction on $\mathcal{M}$, though.

\footnotetext{
${ }^{1}$ A trivial anti-monotonic predicate is one that always evaluates to true
} 
In the algorithms, the alphabet $\Sigma$ being used is restricted to the subset of "interesting symbols", i.e. those symbols that we want to appear in the discovered patterns. Uninteresting or irrelevant symbols are dropped. The algorithms effectively ignores symbols in the database $D$ which do not belong to this restricted $\Sigma$. This deviates a bit from the previous theoretical section. However, in practice, this can significantly prune down the search space, esp. when the original alphabet is very large. This is because, like suffix tries, the size of a VST depends on the alphabet size, which affects the search space of our algorithms. Using a smaller alphabet improves the performance of both algorithms to be introduced.

\subsection{Algorithm VST}

The VST (Version Space Tree) algorithm is based on Agrawal's Apriori [18 algorithm. It consists of two phases:

1. Top-down growing of the version space tree using the anti-monotonic predicate $\mathcal{A}$.

2. Bottom-up marking of the version space tree using the monotonic predicate $\mathcal{M}$.

Both phases are designed to minimise the number of database scans. As such, they both exhibit the cyclic pattern: candidate generation, candidate testing (database scan) and pruning. The cycle terminates when no more new candidates patterns are generated.

Since only the anti-monotonic predicate is handled in phase 1 , we can reuse the idea of Apriori. This is presented as the DESCEND algorithm (Algorithm 1). This algorithm searches the strings satisfying $\mathcal{A}$ in a top-down, breath-first manner. At each depth level $k$ (corresponding to the $k$-th iteration in Apriori), the algorithm first expands the nodes from the previous level. The nodes resulting from expansion is the set $C_{k}$. These candidate nodes are then tested against predicate $\mathcal{A}$. The testing involves one database scan. The candidate patterns that satisfy the predicate are put into $L_{k}$. Those that do not are pruned away from the tree. This is repeated for $k=1,2, \ldots$ until $C_{k}$ is empty. All generated nodes are labelled with $\oplus$ and the suffix links are set up during the process as illustrated in Algorithm 1

Note that the sets $C_{k}$ and $L_{k}$ are the same as the candidate sets and "large" (i.e. frequent) sets in the Apriori algorithm. Moreover, the generation of $C_{k}$ from $L_{k-1}$ also mimics the Apriori-join operation in the Apriori algorithm 2 DESCEND makes use of the suffix link and parent-child relationship of a suffix trie to perform the join efficiently (line 15). The major difference between DESCEND and Apriori is that the former also organizes the discovered strings into a suffix trie, facilitating the join operation and the second phase of the VST algorithm.

\footnotetext{
${ }^{2}$ There are some differences here since we are dealing with strings instead of sets. E.g., while Apriori-join generates generate itemset $\{a, b, c\}$ from $\{a, b\}$ and $\{a, c\}$, the Descend algorithm generates abc from $a b$ and $b c$, because these are the only immediately shorter substrings of abc.
} 


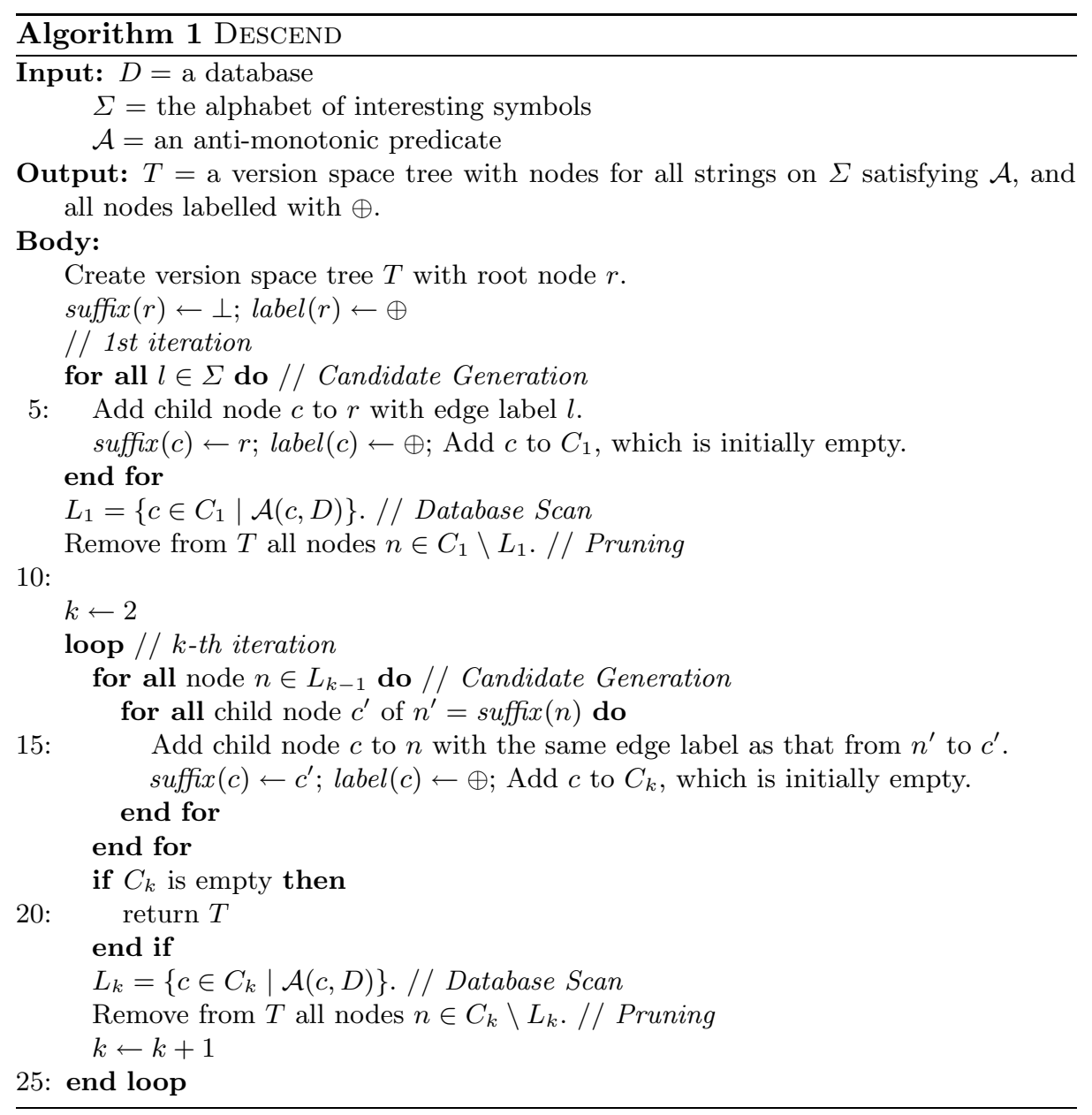




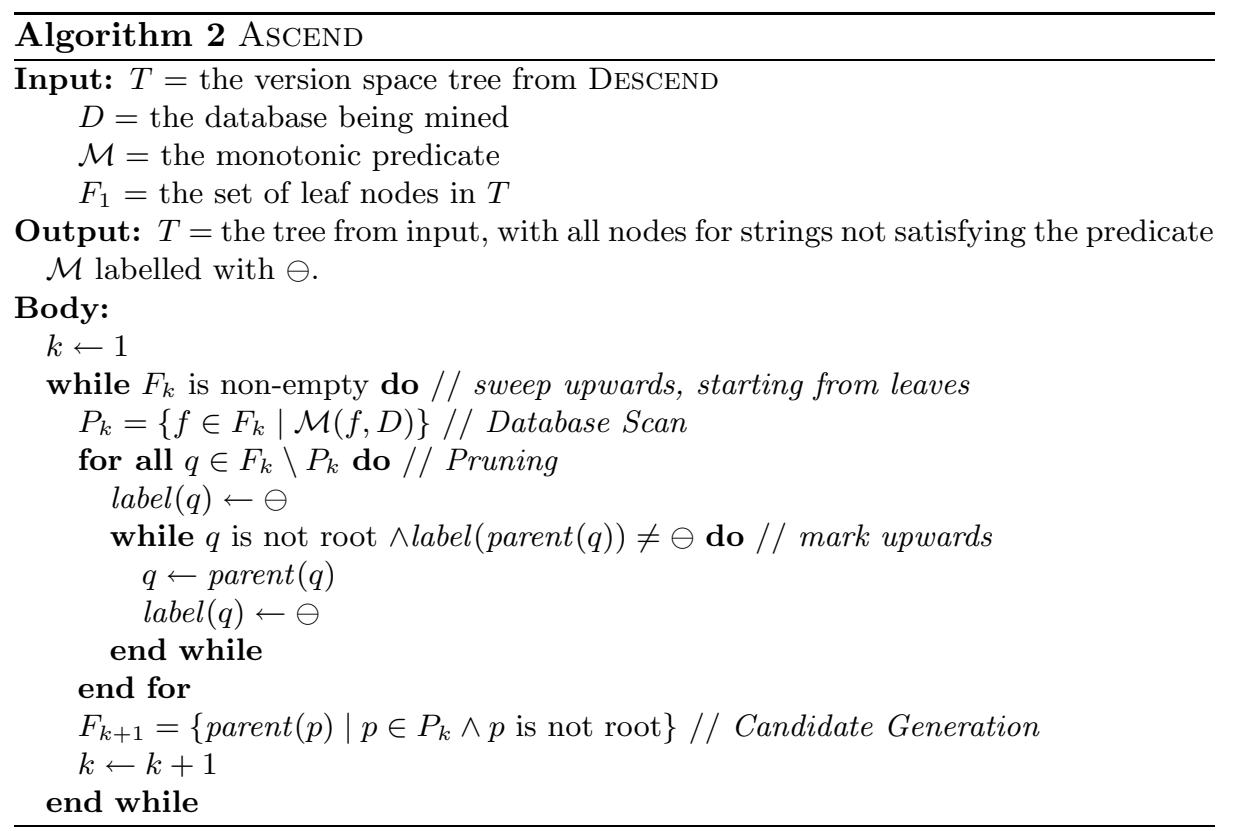

The second phase is implemented with algorithm Ascend. This phase handles the monotonic predicate $\mathcal{M}$. Here we assume that we have the set $F_{0}$ of leave nodes in the tree $T$ generated by DESCEND. $F_{0}$ can be easily obtained by a tree traversal. Actually, it can also be computed during algorithm Descend. While DESCEND works top-down, AsCEND starts from the leaves and works upwards. It first checks the leave nodes against $\mathcal{M}$. If any of these nodes $n$ does not satisfy $\mathcal{M}$, its label is changed to $\ominus$. In addition, all its ancestors are also labelled as $\ominus$, due to the monotonicity. So, we can propagate this $\ominus$ mark upwards until we have marked the root with $\ominus$. Actually, we can stop as soon as we reach an ancestor already marked with $\ominus$, as another such leave node $n^{\prime}$ may share some ancestors with $n$. So, all the ancestors from that point upwards have already been marked with $\ominus$. This is repeated for all $n$ not satisfying $\mathcal{M}$. For nodes $p$ in $F_{0}$ that satisfy $\mathcal{M}$, they should remain labelled $\oplus$. We enter the parent of $p$ into the set $F_{1}$ (with duplication removed), which are to be considered in the next iteration. This is to be repeated until we have an empty $F_{k}$.

So, after these two phases, namely Descend and then Ascend, both $\mathcal{A}$ and $\mathcal{M}$ have been handled. With a simply tree traversal, we can prune away branches which now contains only $\ominus$ children. We have a resulting tree $T$ that is a pruned suffix trie representing all the strings satisfying $\mathcal{P}=\mathcal{A} \wedge \mathcal{M}$.

Theorem 20. The VST algorithm performs at most $2 m$ database scans, where $m$ is the longest string satisfying $\mathcal{A}$.

Proof. The proof is quite straight-forward. Firstly, Descend is just the Apriori algorithm with modifications to handle the suffix trie structure. Therefore, it 
does the same number of database scans as Apriori, which is $m$. For Ascend, we note that it starts with $F_{1}$ containing all the leaves of the resulting $T$ from Descend. So, the deepest one has depth $m$. The $(k-1)$-th iteration of Ascend generates a new $F_{k}$ containing only parents of the previous $F_{k-1}$ (less the pruned ones). As a result, nodes in $F_{k}$ has at most depth $m-k+1$. Since the depth of a non-root nodd 3 must be positive, we have $m-k+1 \geq 1$, i.e. $k \leq m$. Thus, ASCEND makes at most $m$ iterations and hence at most $m$ database scans.

\subsection{Algorithm FAVST}

The drawback of the previous algorithm is that it still has to scan the database $2 m$ times, where $m$ is the length of the longest string satisfying $\mathcal{A}$. Actually, strings exhibit some properties not exhibited by itemsets. Therefore, there is still room for improvements. Our next algorithm, FAVST 4 makes use of techniques from the suffix-tree literature to improve performance. It is well-known in that literature that the suffix-tree of a string can be built in linear time. Some of these ideas are employed in the FAVST algorithm to make it possible to build the version space tree with just a single database scan. We show here only how frequency-based predicates are handled. Database-independent predicates can be handled efficiently without database scanning. For other types of databasedependent predicates, predicate-specific adaptations would be needed.

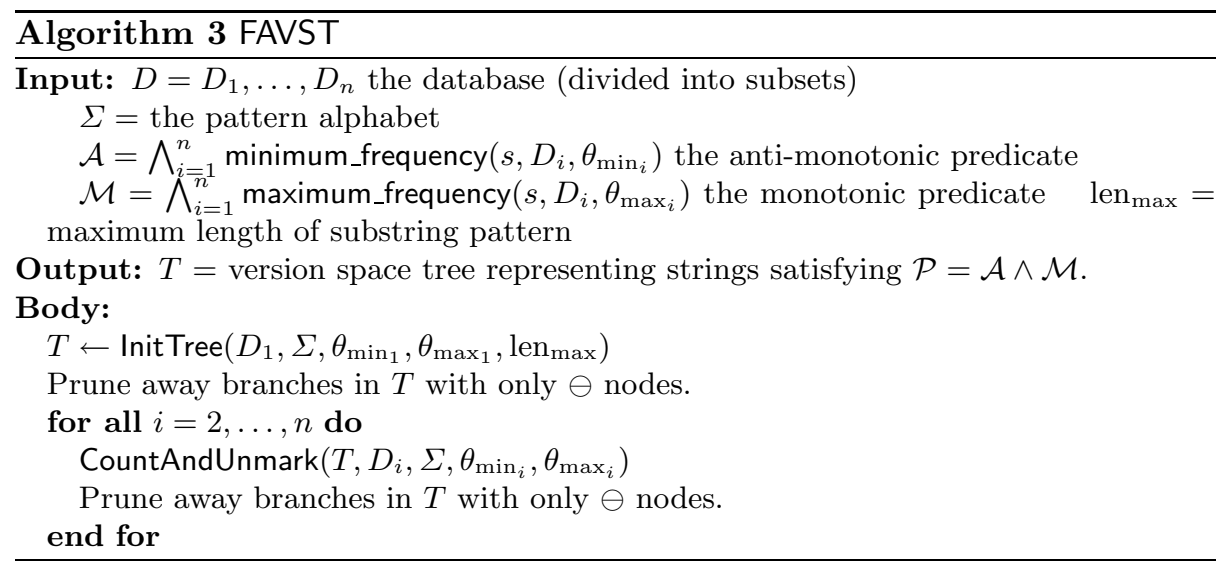

The FAVST algorithm is shown in Algorithm [3. It first calls InitTree to process the first minimum_frequency predicate and scan the first database subset (see Sect. 2.2) to build an initial VST. Then, it invokes CountAndUnmark to process the remaining database subsets and the corresponding minimum_frequency predicates. Note that CountAndUnmark will not grow the VST. It will only count the

\footnotetext{
3 The root node represents the empty string, which needs not be checked against any database.

4 FAVST stands for "Finite Automata-based VST construction".
} 
frequency of the patterns in the corresponding database subset and mark those not satisfying the thresholds $\theta_{\min _{i}}$ and $\theta \max _{i}$ with $\ominus$. Branches with only $\ominus$ are pruned away immediately after the the scanning of each database subset to reduce the number of patterns that need to be checked against the subsequent subsets.

The parameter len $n_{\max }$ specifies an upper bound on the length of the substring patterns to be discovered. When set appropriately, this parameter makes FAVST to be very efficient both in terms of computation time and memory usage (see Sect. (6). FAVST is presented here as if we must specify a minimum and maximum frequency threshold for every database subset. This is indeed not the case. If we are not interested in specify a minimum frequency for subset $i$, we can simply set $\theta_{\min _{i}}=0$. Similarly, a maximum frequency can be set to $\theta_{\min _{i}}=\infty$ to "disable" it. The algorithm in Algorithm 3 is presented in a way to simplify the pseudo-code.

We will later see that each of the subalgorithms InitTree and CountAndUnmark scans the specified database subset only once. So, the whole FAVST algorithm scans each database subset only once. If the database subsets are disjoint, then we can in implementation scan only the subset of data being processed. In that case, FAVST completes in only one scan of the whole database. So, FAVST is a single-scan algorithm.

Algorithm InitTree is shown in Algorithm 4. It scans each string in the database subset symbol for symbol, going down the tree as it proceeds. If a node does not have a suitable child for it to go downward, one such child is created with CreateChild, so that we can go down the tree. Note that this "going down" increases the length of the string pattern represented by the current node $(n)$ by one. To handle the upper bound on the pattern length $\left(\operatorname{len}_{\max }\right)$, the algorithm checks the depth of the current node before actually going down. If the length limit is reached, the algorithm "backs up" by following the suffix link. Essentially, if the current node represents string $a w$ where $a$ is a symbol and $w$ is a string so that $|a w|=\operatorname{len}_{\max }$, then the next substring to be counted would be "aws", which exceeds the length limit. So, we continue with the suffix " $w \varsigma$ ", which is achieved by changing the current node $n$ to $s u f f i x(n)$, because this latter represents string $w$. Next, InitTree increments the count of the destination of this going down, as well as all its suffixes. Then, the next symbol is processed, continuing from this destination. When an uninteresting symbol (i.e. $\notin \Sigma$ ) or the end of a string is encountered, it starts the downward travel from the root node again.

This is basically a suffix tree building algorithm, with four modifications. The first is that we do frequency counting on the way as we go. The second is that we put an upper bound on the length of the substring patterns, or the depth of the trie. Thirdly, we jump back to the root when we encounter uninteresting symbols, saving the need to process any strings containing such symbols. Last but not least, we handle multiple strings, instead of a concatenation of these strings. In the traditional and theoretical approach, multiple strings are handled by building a suffix trie $T_{\text {all }}$ on a single string $s_{1} \$ s_{2} \$ \ldots \$ s_{m}$ (where $m$ is the 


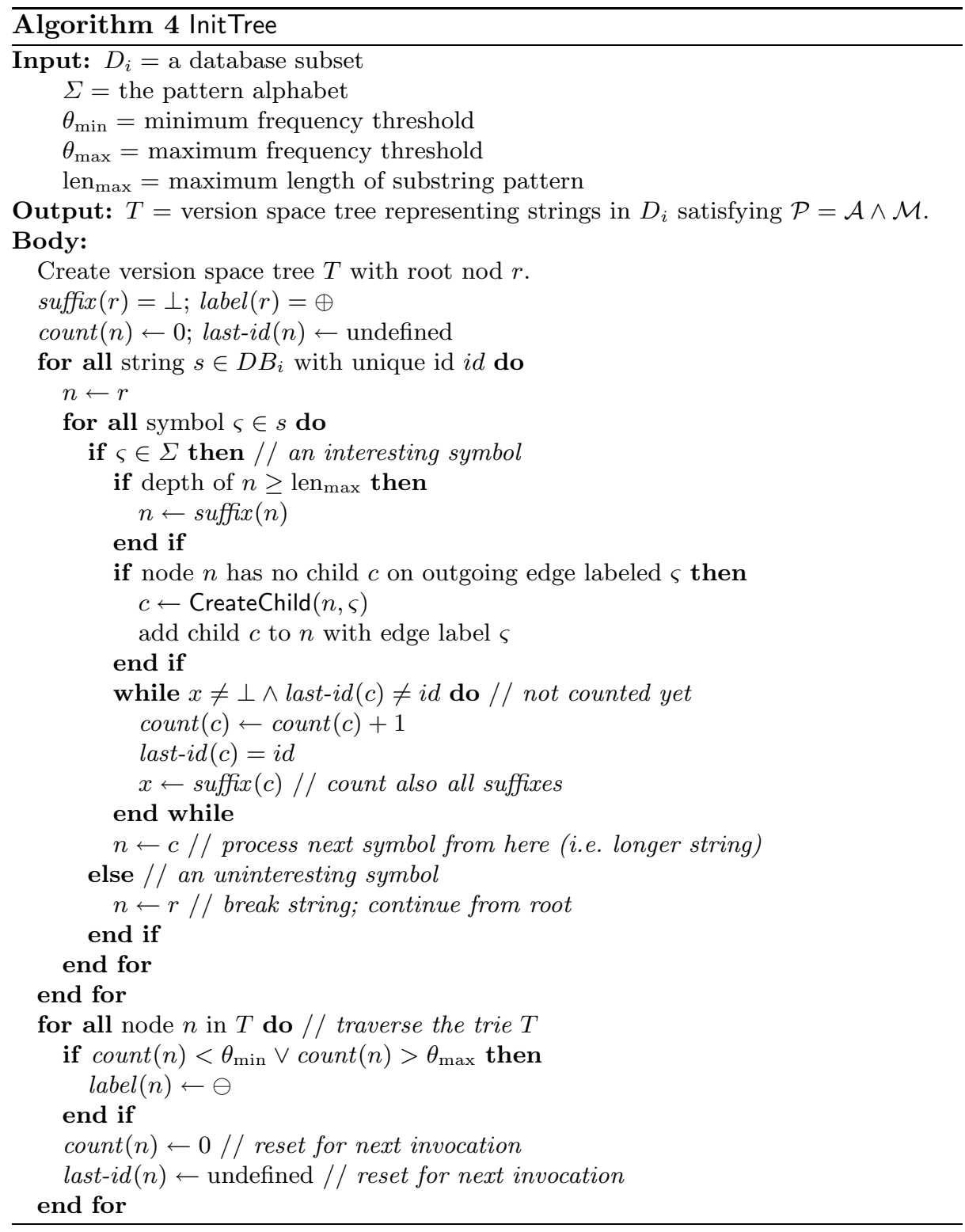


number of strings in $D$ ) obtained by concatenating all strings in $D$, with a special character " $\$$ " not occurring in the database as delimiter. While this approach is a convenient tool for theoretical analysis of complexity, it is costly in implementation as it increases the amount of memory required multifold. It also multiples the depth of the trie, making a complete traversal expensive. Our approach, instead, overlays all the strings $s_{k}$ onto a suffix trie with the same root, maintaining multiplicity with the frequency count $(n)$. Note that our trie $T$ can be obtained by taking $T_{\text {all }}$, cutting it at all the "\$" nodes to obtain a forest of smaller tries, and then merging all these smaller tries at the root to produce a single trie. Uninteresting symbols are handled similarly. Therefore, we have the following theorem.

Theorem 21. The suffix trie $T$ obtained as described above is a sub-trie of the suffix trie $T_{\text {all }}$. Moreover, it takes the same time complexity to build as $T_{\text {all }}$.

After scanning the database, InitTree performs a traversal of the trie and checks if the counts satisfy the specified thresholds. If not, it labels that node with " $\ominus$ ". Meanwhile, the algorithm also resets count $(n)$ and last-id( $n)$ for every node to prepare for the next invocation.

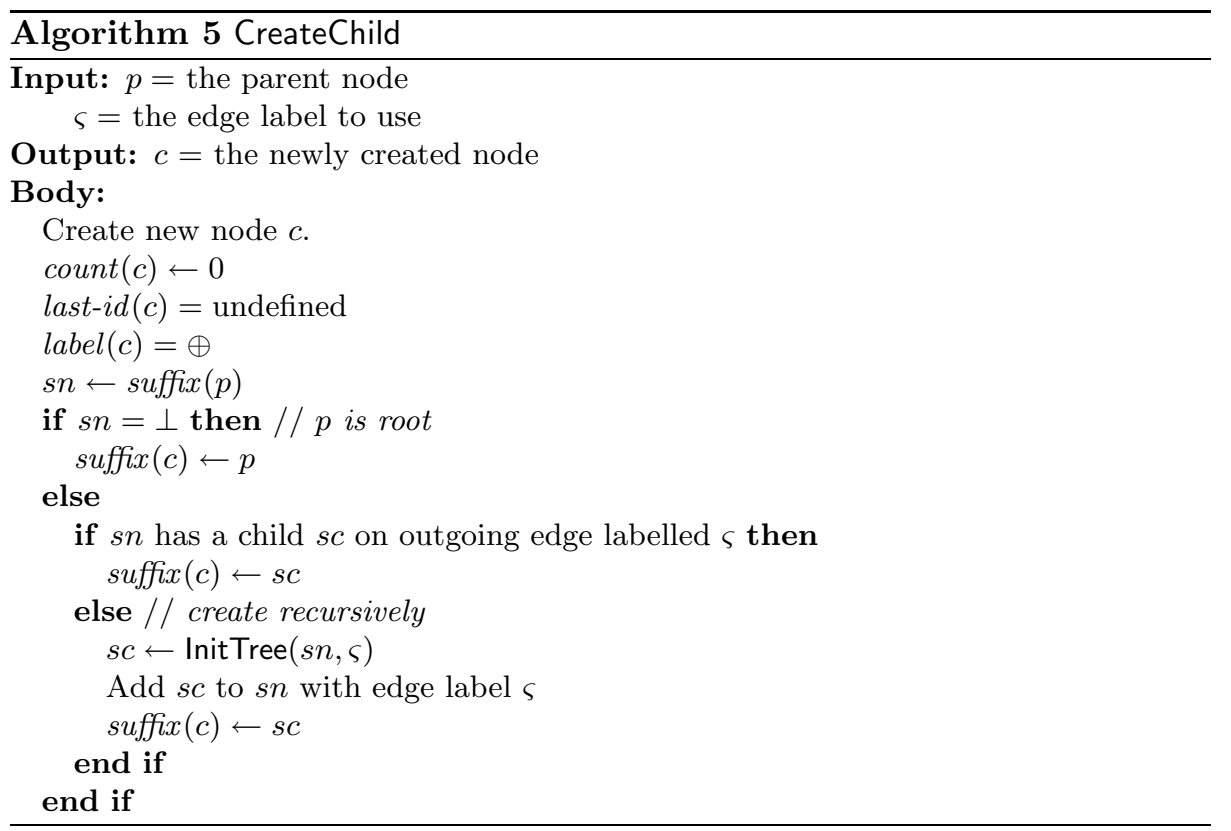

The subroutine CreateChild (Algorithm [5) is relatively straight-forward. It creates and initializes a new node. The most tricky part is to establish the suffix link, recursively creating the node for the suffix if it is not already there. The suffix node can be located by following the parent's suffix. This is because if 
the parent node $p$ represents the string $a w$ (where $a$ is a symbol and $w$ is a string) and the new child node represents string aws, then the suffix of the new child must represent $w \varsigma$, which is represented by a node whose parent represents $w$-the suffix of $p$.

Algorithm 6] show the CountAndUnmark algorithm, which is similar to InitTree except that it does not create new nodes. For any node $n$ already present in $T$, we need not recreate it or re-calculate the suffix link. We only need to increase the support count $\operatorname{count}(n)$ for that node. If the node $n$ is not in $T$ as we do the downward walk, we know that $s$ that it would represent is not present in $T$, and hence it is not a pattern we are looking for (because it doesn't satisfy the predicate $a_{0}$ used to build the initial trie). So, there is no need to create that node. Neither do we need to care about the length limit on the substring patterns, because InitTree has avoided creating the nodes for substrings exceeding the length limit. However, we should continue counting by considering the immediate suffix $s^{\prime}$ of $s$. This is done by following the suffix link of $p$, i.e. $p^{\prime}=\operatorname{suffix}(p)$, which represents the string $t^{\prime}=\operatorname{suffix}(t)$. The node $n^{\prime}$ representing $s^{\prime}$ would be a child of $p^{\prime}$. If it is there, then we have located the node for $s^{\prime}$ and we continue. If not, then we repeat the above method of following suffix links until we have $t^{\prime}=\perp$. The support counts are thus counted in this manner. Again, as we visit a node $n$, we increment the counter on that node to count the occurrence of the corresponding substring pattern.

In order to avoid double-counting a node for the same string 5 in both InitTree and CountAndUnmark, we also record the string id last-id( $n) \leftarrow k$ after incrementing the count. The support count is incremented only if last-id $(n) \neq k$. The nodes are labeled with " $\oplus$ " when they are created.

The major efficiency improvement of FAVST comes from the single database scan. Firstly, note that the algorithm does not work level-wise in the style of Apriori. Rather, it examine the predicates one by one and invokes InitTree and CountAndUnmark to scan the concerned database subsets.

On the space efficiency, since the suffix trie is $O\left(|D|^{2}\right.$ ) in size (where $|D|$ is the total number of symbols in $D$ ), FAVST is less space-efficient than VST. Nevertheless, in practice, we can specify a relatively small upper bound $d$ on the length of the longest substring pattern we are going to find. This can effectively limit the depth of the VST to $d$, reducing the amount of memory that FAVST would need. Of course, a minor modification to FAVST, which is not shown here, is needed.

\footnotetext{
${ }^{5}$ This can happen e.g. for $s_{t}=$ ababc and a node $n$ representing string ab. This is because ab occurs twice in $s_{t}$. However, the frequency is defined in terms of number of strings in $D B_{r}$ containing a string, irrelevant of how many times it occurs in the same string.
} 


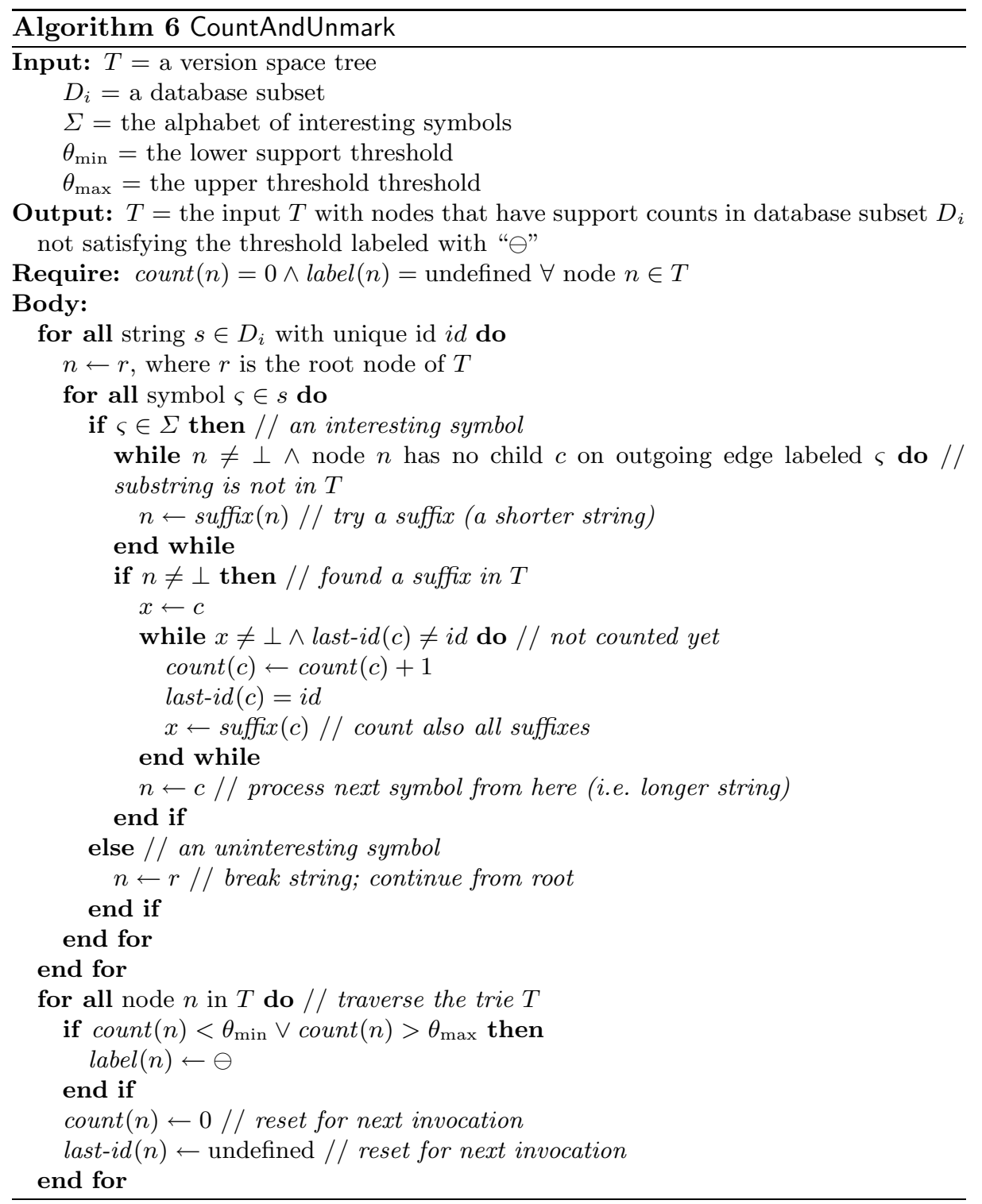




\section{Experiments}

The algorithms VST and FAVST have been implemented in C. The experiments are performed on PC computer with a Pentium-4 2.8GHz processor, 2GB main memory, and running Linux operating system (kernel 2.4.19, glibc 2.2.5).

\subsection{Unix Command History Database}

The database $D B$ used in the experiments are command history collected from 168 Unix users over a period of time. [19] The users are divided into four groups: computer scientists, experienced programmers, novice programmers and nonprogrammers. The corresponding data subsets are denoted "sci", "exp", "nov" and "non", respectively. Each group has a number of users. When each users accesses the Unix system, he first logs in, then type in a sequence of commands, and finally logs out. Each command is taken as a symbol in the database, The sequence of commands from log in to log out constitutes a login session, which is mapped to a string in our experiment. Each user contributes to many login sessions in the database. Table 1 gives some summary data on the database. To study the effectiveness of the len $_{\max }$ parameter to FAVST, we repeated FAVST twice for each experiment: once with $\operatorname{len}_{\max }=\infty$, essentially disabling the length limit; and once with $\operatorname{len}_{\max }=10$. These are denoted "FAVST" and "FAVST"ml", respectively, in all the tables and the figures.

Table 1. Summary statistics of the data

\begin{tabular}{c|c|c|c|c|c|c|c}
$\begin{array}{c}\text { Subset } \\
\left(D_{i}\right)\end{array}$ & no. of & no. of & $\theta_{\text {min }}$ & frequent & \multicolumn{3}{|c}{ time (milliseconds) } \\
\cline { 5 - 8 } & strings & & substrings & VST & FAVST & FAVST \\
\hline nov & 55 & 5164 & 24 & $294^{\star}$ & 770 & 1040 & 330 \\
exp & 36 & 3859 & 80 & 292 & 700 & 950 & 530 \\
non & 25 & 1906 & 80 & 293 & 180 & 280 & 110 \\
sci & 52 & 7751 & 48 & 295 & 1170 & 2310 & 1010
\end{tabular}

${ }^{\star}$ Of these 294 patterns, 36 have length $>10$. They are thus dropped in FAVST ${ }^{\mathrm{ml}}$.

\subsection{Performance - minimum_frequency only}

The first set of experiments are done with only one minimum_frequency predicate. The thresholds used are shown in Table 1. These thresholds are selected so that they produce around 300 frequent string patterns in each database subset. The time taken (wall-clock time) by the two algorithms are noted and given in the same table.

It is promising that FAVST ${ }^{\mathrm{ml}}$ is the fastest in all cases. With an upper bound on the length of the substring patterns, FAVST works much more efficiently, because of the reduction of the size of the trie structure. The drawback is that 36 patterns are pruned away by this length limit. 
On the other hand, it seems disappointing that FAVST takes longer time to finish than VST, despite our claim of a single database scan. Our explanation is that the data files are stored in local harddisk, and hence are extremely fast to access. Thus, the single-scan advantage of FAVST is suppressed. Moreover, the disk caching effect also diminishes the advantage of a single database scan. However, this is only valid for small databases which can be accessed quickly (e.g. local drive). Much larger databases that do not fit into main memory or that are stored on much slower storage devices (e.g. on CDROM, on a file server accessed via a LAN, or even over a web server) can benefit from the single database scan. To simulate this idea, we modified the programs to introduce a delay in the database reading routine. The delay is parameter-controlled. It inserts $m$ milliseconds of delay for every 100 transactions (i.e. strings) read. Repeating the above experiment with different values of $m$ gives the results in Figure 2. We plot the result of each data set separately, comparing the two algorithms in each case.

Novice programmers (nov)

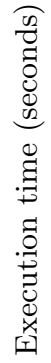

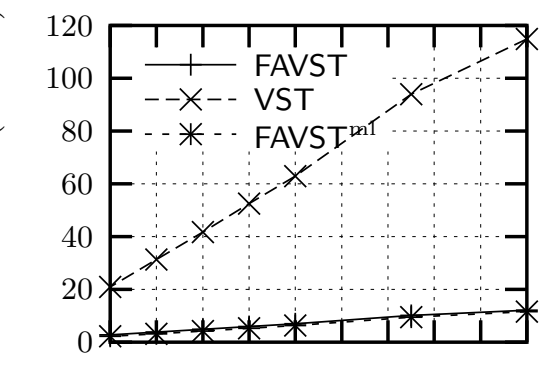

102030405060708090100

Artificial Delay (ms/100 transactions)

Non-programmers (non)

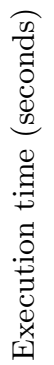

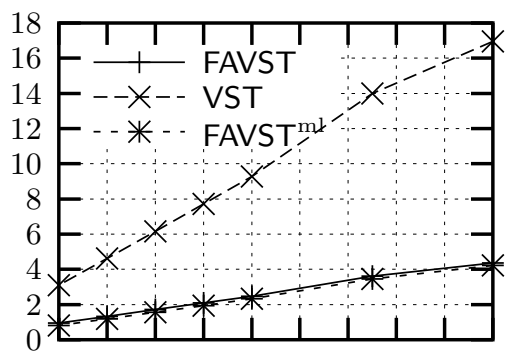

$10203040506070 \quad 8090100$

Artificial Delay (ms/100 transactions)

Expert programmers (exp)

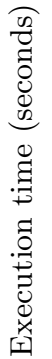

$\begin{array}{lllllllll}10 & 20 & 30 & 40 & 50 & 60 & 70 & 80 & 90100\end{array}$

Artificial Delay (ms/100 transactions)

Computer Scientists (sci)

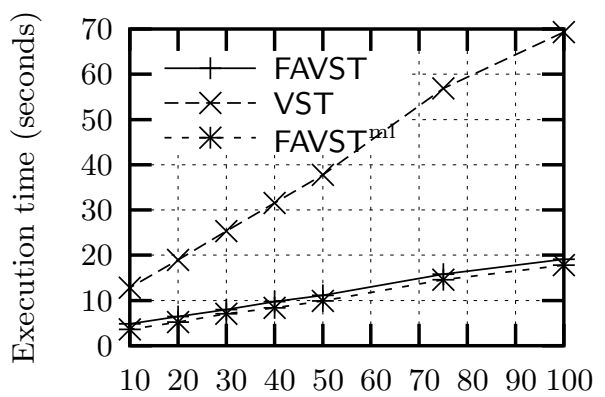

Artificial Delay (ms/100 transactions)

Fig. 2. Performance comparison with database access delays 
It is clear from the figure that FAVST out-performs VST once the database access delay becomes non-trivial. The former is consistently 3 times faster than the latter. For the "nov" data set, the speed up ratio even reaches 6. Evidently, the single database scan algorithm FAVST scales much better with large database with slow access than the simpler algorithm VST. The effect of the limit on pattern length does not appear significant here. The curves for FAVST and FAVST ${ }^{\mathrm{ml}}$ almost overlap for all four data sets. This is because the dominant factor in execution time is the number of database scans, which is always 1 , whether or not we specify a length limit on the patterns.

\subsection{Memory Footprints}

The speed up is a trade off with memory usage. Table 2 shows the maximum amount of memory consumed by the two algorithms for the data structures. VST has a memory footprint in the order of tens of kilobytes, whereas that of FAVST is in megabytes. With today's computing equipments, the memory consumption of FAVST is absolutely affordable. Imposing a limit on the length of patterns makes FAVST ${ }^{\mathrm{ml}}$ build a much smaller trie than FAVST, significantly reducing the memory consumption by a factor of $2-5$.

Table 2. Memory usage of the two algorithms

\begin{tabular}{c|c|c|c|c|c} 
Data sub- & \multicolumn{3}{|c|}{ Max. mem. usage (bytes) } & \multicolumn{2}{c}{ Ratio } \\
\cline { 2 - 6 } set $\left(D_{i}\right)$ & VST & FAVST & FAVST $^{\mathrm{mI}}$ & FAVST/VST & FAVST $^{\mathrm{ml}} /$ VST $^{-}$ \\
\hline nov & 57158 & 17456767 & 3573695 & 305 & 63.5 \\
$\exp$ & 88870 & 14439604 & 5367732 & 162 & 60.4 \\
non & 59918 & 6797462 & 2081558 & 113 & 34.7 \\
sci & 94454 & 23107503 & 8997455 & 245 & 95.3
\end{tabular}

It should be noted that the memory consumption of the algorithms has no direct relation to the database sizes. From Table 1, we can see that the data set "nov" is larger than "exp" and "non". However, it turns out to cause the algorithms to consume less memory than the other two data sets. Our explanation is that the "nov" data set has more repeated string patterns. Since our algorithm uses the same trie node for the same pattern, the fewer the number of distinct string patterns, the fewer nodes are created, and hence the less memory consumed. In other words, the memory consumption is related to the number of distinct string patterns, but not the database size. Thus, our algorithms exhibits very nice properties for data mining applications. They scale well with database size, and use an amount of memory depending on the amount of interesting patterns that will be discovered.

\subsection{Performance-Compound Predicates}

The above experiments only makes use of the minimum frequency predicate. No monotonic predicates are specified. Thus, the full features of our algorithms has 
not been utilized. The following experiment uses the algorithms VST and FAVST to compute two version space trees $T_{1}$ and $T_{2}$, each representing a set of strings satisfying a predicate of the form $\mathcal{P}=\mathcal{A} \wedge \mathcal{M}$, where $\mathcal{A}$ is minimum_frequency and $\mathcal{M}$ is maximum_frequency. The details are tabulated in Table 3. The database used is the same as described above. The thresholds for the minimum frequency predicate are copied from Table [1, where as those for the maximum_frequency are from Table 1 less 25\%. After computing these trees, the union of them, $U$ is computed by a naïve tree-merging operation. Note that $U$ is no longer a version space tree, as it represents a subsets of $\Sigma^{*}$ which is not a version space anymore.

Table 3. An experiment on compound predicates

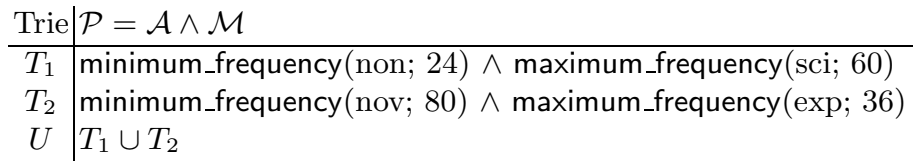

The results of the experiments are shown in Table 4. Each row shows the time that either algorithm used to build that tree. The time taken to compute $U$ in either case is negligible, as it is done completely in memory. It takes so little time (less than 0.01 second) that we cannot reliably measure because of the granularity of the time-measurement program we are using.

Table 4. Results on finding the union of two version spaces

\begin{tabular}{c|c|c|c|c|c|c}
\multirow{2}{*}{ Trie } & \multicolumn{2}{|c|}{ Time (seconds) } & \multicolumn{3}{c}{ number of nodes } \\
\cline { 2 - 6 } & VST & FAVST & FAVST & labeled $\oplus$ & labeled $\ominus$ & total \\
\hline$T_{1}$ & 0.56 & 0.39 & 0.19 & 166 & 40 & 206 \\
$T_{2}$ & 1.23 & 1.19 & 0.37 & $237^{\star}$ & 18 & 255 \\
\cline { 2 - 3 }$U$ & \multicolumn{2}{|c|}{ negligible } & $401^{\star}$ & 47 & 448
\end{tabular}

${ }^{\star}$ Of these $\oplus$ nodes, 36 are at depth $>10$, representing patterns of length $>10$. These are pruned away in the FAVST ${ }^{\mathrm{ml}}$ case.

It is encouraging that in this general case, FAVST runs faster than VST, even though we did not add the artificial database access delay into the programs. FAVST $^{\mathrm{ml}}$ takes even less time to compute the result, although 36 patterns are not discovered due to the length limit.

The longest pattern found (represented by the deepest node in $U$ having $a \oplus$ label) was "pix umacs pix umacs pix umacs pix umacs pix umacs pix umacs pix umacs pix umacs pix umacs pix", which has a length of 19 . The deepest $\ominus$ node in $U$ represented the string "cd ls cd 1s", of length 4, which has an interesting (labelled $\oplus$ ) child representing the string "cd 1 s cd Is e". If we did not have any monotonic predicates (i.e. maximum_frequency in this case), "cd ls cd ls" would have been considered interesting because it is frequent 
enough. However, with the monotonic predicates, this string is now too frequent and hence it is considered uninteresting and marked with $\ominus$ in $U$. This illustrates the increased power and expressiveness of using both anti-monotonic and monotonic predicates together in data mining. The ability to compute $U$ efficiently by manipulating the results $T_{1}$ and $T_{2}$ shows the power of these algorithms in combination with the results in 609.

\section{Conclusions}

In this paper, we have addressed the problem of Mining Substring Patterns under conjunctive constraints. This is a core component of a more general data mining framework in a couple of related works 6]910.

The main contribution of this paper was the FAVST algorithm, which employs the VST data structure of 69 and combines principles of constraint based mining with those of suffix-trees. This algorithm has the very nice property of requiring only one database scan, at the expense of very affordable memory overheads. Such overheads can be significantly reduced by imposing an upperbound on the length of patterns. The data structure and algorithms have been empirically proved to be practical and useful for finding substring patterns in a unix user command database.

One direction for further research is concerned with data streams. It might be possible to combine the present framework with that proposed by Han et al.

\section{References}

1. Creighton, C., Hanash, S.: Mining gene expression databases for association rules. Bioinformatics 19 (2003) 79-86

2. Agrawal, R., Imielinski, T., Swami, A.N.: Mining association rules between sets of items in large databases. In Buneman, P., Jajodia, S., eds.: Proceedings of the 1993 ACM SIGMOD International Conference on Management of Data, Washington, D.C., U.S.A. (1993) 207-216

3. Agrawal, R., Srikant, R.: Mining sequential patterns. In Yu, P.S., Chen, A.S.P., eds.: Eleventh International Conference on Data Engineering, Taipei, Taiwan, IEEE, IEEE Computer Society Press (1995) 3-14

4. Ukkonen, E.: On-line construction of suffix trees. Algorithmica 14 (1995) 249-260

5. Weiner, P.: Linear pattern matching algorithm. In: Proc. 14 IEEE Symposium on Switching and Automata Theory. (1973) 1-11

6. De Raedt, L., Jaeger, M., Lee, S.D., Mannila, H.: A theory of inductive query answering (extended abstract). In Kumar, V., Tsumoto, S., Zhong, N., Philip S. Yu, X.W., eds.: Proc. The 2002 IEEE International Conference on Data Mining (ICDM'02), Maebashi, Japan (2002) 123-130 ISBN 0-7695-1754-4.

7. Kramer, S., De Raedt, L., Helma, C.: Molecular feature mining in HIV data. In: KDD-2001: The Seventh ACM SIGKDD International Conference on Knowledge Discovery and Data Mining, Association for Computing Machinery (2001) ISBN: 158113391X. 
8. De Raedt, L., Kramer, S.: The levelwise version space algorithm and its application to molecular fragment finding. In: IJCAI01: Seventeenth International Joint Conference on Artificial Intelligence. (2001)

9. De Raedt, L., Jaeger, M., Lee, S.D., Mannila, H.: A theory of inductive query answering. (2003) (submitted to a journal).

10. Lee, S.D., De Raedt, L.: An algebra for inductive query evaluation. 20 147-154

11. Grahne, G., Lakshmanan, L.V.S., Wang, X.: Efficient mining of constrained correlated sets. In: Proceedings of the 16th International Conference on Data Engineering, IEEE Computer Society (2000) 512-521

12. Han, J., Pei, J., Yin, Y.: Mining frequent patterns without candidate generation. In Chen, W., Naughton, J.F., Bernstein, P.A., eds.: Proceedings of the 2000 ACM SIGMOD International Conference on Management of Data, Dallas, Texas, U.S.A., ACM Press (2000) 1-12

13. Pei, J., Han, J.: Can we push more constraints into frequent pattern mining? In: Proceedings of the Sixth ACM SIGKDD International Conference on Knowledge Discovery and Data Mining (KDD 2000), Boston, MA, USA (2000) ISBN: 1-58113233-6.

14. Fischer, J., De Raedt, L.: Towards optimizing conjunctive inductive queries. In: Proc. The Eighth Pacific-Asia Conference on Knowledge Discovery and Data Mining (PAKDD2004), Carlton Crest Hotel, Sydney, Australia (2004)

15. Boulicaut, J.F., Jeudy, B.: Using constraints during set mining: Should we prune or not? In: Actes des Seizième Journées Bases de Données Avancées (BDA'00), Blois, France (2000) 221-237

16. Bonchi, F., Giannotti, F., Mazzanti, A., Pedreschi, D.: ExAMiner: Optimized level-wise frequent pattern mining with monotone constraints. 20 11-18

17. Mitchell, T.M.: Generalization as search. Artificial Intelligence 18 (1982) 203-226

18. Agrawal, R., Srikant, R.: Fast algorithms for mining association rules. In Bocca, J.B., Jarke, M., Zaniolo, C., eds.: Proceedings of the 20th International Conference on Very Large Databases, Santiago, Chile, Morgan Kaufmann (1994) 487-499

19. Greenberg, S.: Using unix: Collected traces of 168 users. Research Report $88 / 333 / 45$, Department of Computer Science, University of Calgary, Alberta, Canada. (1988)

20. Wu, X., Tuzhilin, A., Shavlik, J., eds.: Proceedings of The Third IEEE International Conference on Data Mining (ICDM'03). In Wu, X., Tuzhilin, A., Shavlik, J., eds.: Proceedings of The Third IEEE International Conference on Data Mining (ICDM'03), Melbourne, Florida, USA, Sponsored by the IEEE Computer Society (2003) 